\title{
DIABETES
}

\section{Does glycemic control reduce cardiovascular event rates?}

Controlling blood glucose to normal fasting levels with insulin glargine or adding n-3 fatty acids has a neutral effect on cardiovascular outcomes in patients with dysglycemia. The data come from the Outcome Reduction with an Initial Glargine Intervention (ORIGIN) trial.

This study was an international, randomized, 2-by-2 factorial trial with comparisons of both open-label insulin glargine and double-blind n-3 fatty acid treatment. The investigators enrolled $>12,500$ individuals (mean age 63.5 years) with impaired glucose fasting, impaired glucose tolerance, or type 2 diabetes mellitus, and who were at high risk of cardiovascular events. Individuals were then randomly allocated to receive standard care with or without the addition of a subcutaneous, titrated injection of insulin glargine to target the fasting blood glucose level to $\leq 5.3 \mathrm{mmol} / \mathrm{l}$. Additionally, they were randomly allocated to receive a daily capsule containing $1 \mathrm{~g}$ of either $\mathrm{n}-3$ fatty acids (465 mg eicosapentaenoic acid and $375 \mathrm{mg}$ docosahexaenoic acid) or

\section{1 ...the findings of the ORIGIN trial do not support changing standard therapies... 77}

olive oil as a placebo. The median duration of follow-up was 6.2 years.

The rate of nonfatal myocardial infarction, nonfatal stroke, or death from cardiovascular causes was not different with insulin-glargine treatment compared with standard care (2.94 versus 2.85 per 100 person-years; HR 1.02, 95\% CI 0.94-1.11, $P=0.63$ ), and remained not different when revascularization or hospitalization for heart failure were added to the composite outcome. Insulin glargine seemed to reduce the rate of incident diabetes, but increase the likelihood of hypoglycemia or modest weight gain.

At the end of follow-up, triglyceride levels were $0.16 \mathrm{mmol} / \mathrm{l}$ lower in patients receiving $n-3$ fatty acids than in those taking placebo $(P<0.001)$, but mortality from cardiovascular causes did not differ between the groups (9.1\% versus 9.3\%; HR $0.98,95 \%$ CI $0.87-1.10, P=0.72$ ). The rates of major vascular events, death from any cause, or death from arrhythmia were also not different between the groups.

The researchers conclude that "the findings of the ORIGIN trial do not support changing standard therapies for early dysglycemia". They note that insulin glargine seems to slow the progression of dysglycemia, and that outcome rates might have been confounded by background metformin use. The potential beneficial effects of doses of n-3 fatty acids $>1 \mathrm{~g}$ are unknown, and the findings of this study should not affect "dietary recommendations to consume more fish".

Gregory B. Lim

Original articles The ORIGIN trial investigators.
Basal insulin and cardiovascular and other outcomes
in dysglycemia. N. Engl. J. Med. doi:10.1056/
NEJMoa1203858 | The ORIGIN trial investigators.
n-3 Fatty acids and cardiovascular outcomes in patients
with dysglycemia. N. Engl. J. Med. doi:10.1056/
NEJMoa1203859

